\title{
Programa de orientación psicopedagógico de orientación cognitiva en la mejora de la competencia matemática
}

\author{
Heydi Tananta Vásquez \\ htananta@ucv.edu.pe \\ Carmen Jianina García Córdova \\ cordovaj@ucvvirtual.ed.pe \\ garciacord1993@hotmail.com \\ Angie Milagros Panduro Vela \\ Angiemilagros_28@hotmail.com \\ Universidad César Vallejo \\ Tarapoto - Perú
}

\section{RESUMEN}

La competencia matemática hace referencia a la habilidad para poder utilizar y asociar los números, sus operaciones básicas, los símbolos y formas de expresión numérica, estos están muy relacionados con aspectos de pensamiento matemático, planteamiento y resolución de problemas, análisis y diseño de modelos, representaciones y sobre todo comunicación sobre las matemáticas. En ese sentido, el presente artículo tiene como objetivo establecer la eficacia de un Programa de intervención psicopedagógico de orientación cognitivo en la mejora de la competencia matemática, para lo cual se seleccionó una muestra de 50 estudiantes del 3er grado de primaria de una institución educativa del Distrito de la Banda de Shilcayo. El diseño de investigación es experimental de tipo pre-experimental, en ese sentido, se aplicó un pre y post test, para los resultados utilizamos contraste estadístico de T de Student para muestras emparejadas, así se obtuvo que tanto en la dimensión cálculo y numeración el p-valor $(0,000)$ está por debajo del nivel de riesgo (5\%), por tanto, se obtuvo como resultado que el programa de intervención psicopedagógico de orientación cognitivo es eficaz en la mejora de la competencia matemática.

Palabras clave: competencias; programas; orientación, aprendizaje, cognitivo. 


\title{
Psychopedagogical intervention program of cognitive orientation in the improvement of mathematical competence
}

\begin{abstract}
Mathematical competence refers to the ability to use and associate numbers, their basic operations, symbols and forms of numerical expression, these are closely related to aspects of mathematical thinking, posing and solving problems, analysis and design of models, representations and especially communication about mathematics. In this sense, the present article aims to establish the efficacy of a cognitive-oriented psychopedagogical intervention program in improving mathematical competence, for which a sample of 50 students of the 3rd grade of primary school from an educational institution of the Shilcayo Band District. The research design is experimental of a preexperimental type, in that sense, a pre and posttest was applied, for the results we used statistical contrast of Student's t for paired samples, thus it was obtained that both in the calculation dimension and numbering the p-value (0.000) is below the risk level (5\%), therefore, it was obtained as a result that the cognitive-oriented psychopedagogical intervention program is effective in improving mathematical competence
\end{abstract}

Keywords: competences; programs; orientation, learning, cognitive

Artículo recibido: 02 noviembre. 2021 Aceptado para publicación: 28 noviembre 2021 Correspondencia: htananta@ucv.edu.pe Conflictos de Interés: Ninguna que declarar 


\section{INTRODUCCIÓN}

La educación es el proceso de socialización, formación teórica y práctica de los seres humanos en crecimiento y desarrollo, en el cual se suministran herramientas y técnicas de conocimientos esenciales para poner en práctica a lo largo de nuestras vidas, en este sentido, es importante incluir dentro de ello al aprendizaje, el mismo que nos va a permitir adquirir habilidades, destrezas, conocimientos y conductas a lo largo de nuestras vida, pues cabe resaltar que, durante todo el proceso de nuestras vidas vamos aprendiendo desde el hecho de encontrar las primeras formas de comunicación y resolución de problemas de acuerdo a etapas en las que nos encontramos hasta nuestro último día de vida, pues ello nos permite modificar conductas, ganar experiencias, razonar y observar nuestras conductas (Rodríguez, 2014).

En este sentido, es importante mencionar que la Educación Básica Regular está orientada a beneficiar el desarrollo integral del estudiante, el acrecentamiento de sus potencialidades y el incremento de capacidades, conocimientos, actitudes y valores primordiales que la persona debe tener para actuar adecuada y eficazmente en los diversos ámbitos sociales (Ruiz, 2011). Sin embargo, observamos que, durante todo este proceso, existen dificultades que deben ser consideradas al momento de generar un conocimiento, pues el proceso de aprendizaje y educación se vuelve más lenta y es preciso crear o mejorar nuevas formas de enseñanza que permitan al educando la adquisición de conocimientos (Carrillo, 2009).

Además, debemos resaltar que la educación de un individuo conlleva a un continuo proceso de transformación y búsqueda de perfección, el mismo que debe estar articulada a las condiciones sociales y culturales históricas en el que se desarrollen los individuos. Es desde ahí que las personas accedemos a la enseñanza y/o aprendizaje de las ciencias, toda vez que pueda participar y aprender de los discursos científicos enriqueciendo e incorporando a nuestra formación cognitiva, afectiva, volitiva, ética y política. La calidad del proceso de incorporación individual de la cultura es esencial en la formación y el desarrollo de competencias "verdaderas" en la escuela.

Es así que podemos hacer mención que, los seres humanos, inician su aprendizaje numérico a los 2 años, empiezan con el uso de la palabra "dos" a fin de alegar a todas las pluralidades: pasado los 2 años utilizan "tres" para asignar "varias cosas", les ofrece la facilidad de rotular recopilaciones con números, tienen una representación puntual de fijar 
"igual", "diferente" o "más". Describir brinda a los seres humanos la relación entre lo que observa de forma directa y las consignas matemáticas abstractas. (León et al., 2014)

Cerda, et al. (2011) Refiere que la educación del nivel inicial, la competencia corresponde al hecho de que el niño(a) manipule objetos matemáticos, fomente la creatividad, se dé cuenta y evalúe su proceso de pensamiento, coge confianza en sí mismo, se entretenga con su propia actividad mental, asocie y diferencie con otros escenarios de su vida cotidiana y se prepare para nuevos retos tecnológicos. En la etapa pre escolar e inicial la manipulación de objetos es primordial para que los niños (as) realicen las conceptualizaciones correspondientes al pensamiento matemático, además es preciso indicar que las atmosferas propuestas posibiliten la transferencia del saber considerando la resolución de problemas y el uso del saber.

Por otro lado, una de las áreas de nivel primario que para muchos estudiantes resulta complejo de lograr es precisamente las competencias matemáticas, definidos en palabras como la habilidad para poder utilizar y asociar los números, sus operaciones básicas, los símbolos y formas de expresión numérica, estos están muy relacionados con aspectos de pensamiento matemático, planteamiento y resolución de problemas, análisis y diseño de modelos, representaciones y sobre todo comunicación sobre las matemáticas.

Bajo este contexto, entonces hacemos mención que, el significado de competencia que consiguió la educación formal, inició en el campo del lenguaje a través de la competición y lingüística la competencia comunicativa. Este aporte se complementó con la hipótesis del procesamiento de investigación, competencias laborales e inteligencias múltiples, direccionando el significado de competitividad a otros cursos de educación por competencias matemáticas, competencias comunicativas, etc. (Tobón, 2008)

Entonces, Interpretar las matemáticas como un lenguaje implica que los estudiantes deben reconocer las señales organizados que se presentan en las disertaciones matemáticas como, los términos, los signos o símbolos para identificar los clasificaciones y destrezas que vamos a generar con algunas operaciones matemáticas, dar respuesta a problemas en diversos contextos (OCDE, 2006).

En la relación al pensamiento y lenguaje, Vigostky (1982) refiere que el desarrollo del pensamiento está influenciado fuertemente por el lenguaje, es decir juegan un papel muy importante las herramientas lingüísticas del pensamiento, así como de la experiencia social y cultural del niño, además cabe resaltar que el desarrollo intelectual del niño(a) 
depende del dominio de los medios sociales del pensamiento. Además, las capacidades no solo se miden en conocimientos, habilidades y hábitos, sino en la dinámica del proceso del como adquirir, es decir, cuán rápido, profundo, fácil y constante resultad el proceso individual para lograr un dominio de conocimientos y habilidades fundamentalmente importante para algunos tipos de actividades. Entonces es donde se ponen en evidencia las diferencias para hablar de las capacidades (Petrovski, 1986)

Por todo lo mencionado, entonces debemos referir que la enseñanza y el aprendizaje de las matemáticas son procesos sociales de progresiva complejidad teórica y metodológica. Por un lado, la didáctica de las matemáticas tuvo un creciente avance hasta convertirse en una disciplina científica (Gascón, 1998) y por lo cual, direcciona su objeto de estudio en alrededor de su propósito fundamental: estudiar científicamente problemas de enseñanza y aprendizaje de las matemáticas en contextos educativos institucionalizados. Por otro lado, las separaciones epistemológicas y ontológicas en las matemáticas y en su didáctica, indican replanteamientos sobre el rol del docente y estudiante, sobre la enseñanza del área y sobre su desarrollo didáctico y curricular.

En este proceso, la interacción y la intersubjetividad de los sujetos que construyen y reconstruyen sus representaciones son esenciales para hacer posible la enseñanza y el aprendizaje de calidad y, en consecuencia, lógica para asumir en el marco de la didáctica de las matemáticas, su complejo proceso de formación y desarrollo (García et ál., 2009) Por otro lado, los argumentos disciplinarios de las matemáticas, en contraposición a un currículo centrado en contenidos, se enseñan con la finalidad de gestionar la cimentación de conocimientos matemáticos contextualizados, direccionados a mayores niveles de conceptualización y/o abstracción, así como de la construcción de complejidad a través de estímulos de formación que propicien el desarrollo de pensamiento matemático y potencie la formación de competencias matemáticas. Las competencias suelen trascender por su naturaleza dinámica y compleja de la propia disciplina. En consecuencia, pensar en las competencias matemáticas como medio y fin en sí mismas en la práctica educativa resulta fundamental para poder aprender matemática (García et ál., 2009).

En consecuencia, estos conceptos se podrían asumir que el conocimiento matemático no es una réplica objetiva de una sola realidad ajena al individuo, sino una cimentación personal y social de consideraciones, además de ser un resultado del progreso histórico, 
un desarrollo permanente de tipo cultural, situado en contextos específicos (D'Amore, Godino y Fandiño, 2008).

La Organización para la Cooperación y el Desarrollo Económico (OCDE) realiza cada tres años el Programa Internacional para la Evaluación de Estudiantes (PISA, por sus siglas en inglés) dirigido a estudiantes de 15 años. En la evaluación PISA 2015 aplicado a 72 países, 9 de los cuales fueron de Latinoamérica, se buscó medir las competencias en: lectura, matemática, ciencia, educación financiera y resolución de problemas colaborativos. En el Perú fueron evaluados 281 instituciones educativas de todas las regiones del país, tanto públicas como privadas, urbanas y rurales. Así, los resultados indican que Perú tuvo una mejora significativa respecto a la evaluación PISA 2012, dejando el último lugar de la tabla de resultados y resaltando mejoras en tres materias (ciencia, matemática y lectura) (Ministerio de Educación [MINEDU], 2017).

A pesar del logro obtenido en los resultados PISA 2015 logrando salir de la última posición, el Perú aún mantiene un sistema educativo con baja calidad educativa sobre todo en el área de matemática y comprensión lectora, problema que converge en los resultados de las evaluaciones internacionales como es el caso de PISA. Siendo PISA una de las principales evaluaciones a gran escala, cuyo objetivo es evaluar hasta dónde los estudiantes de 15 años que se encuentran en nivel secundario tanto de escuelas públicas y privadas utilizan sus conocimientos y habilidades necesarios para afrontar situaciones y desafíos que son planteadas por la sociedad actual (MINEDU, 2017).

Los resultados de PISA 2015 presentan similar resultado respecto a la Evaluación Censal de Estudiantes (ECE) 2016 realizado por el MINEDU que definen una escala de medición de tres niveles (en inicio, en proceso y satisfactorio). La evaluación se realiza en las áreas de matemática y lectura dirigido a estudiantes de $2^{\circ}$ grado de primaria, $2^{\circ}$ grado de secundaria, $4^{\circ}$ grado de primaria y $4^{\circ}$ grado de secundaria tuvo como resultado de logro inicio y proceso con un bajo nivel de resultado satisfactorio. Resultados que reafirman que la calidad educativa en Perú necesita una urgente intervención y sobre todo un análisis de las causas para realizar propuestas que contribuyan a la mejorar la calidad educativa y por ende la competencia matemática (MINEDU, 2016).

Dockrell y McShane (citado por Ortega, 2014) consideran que las dificultades de aprendizaje son aquellas que obstaculizan el éxito educativo, y el nivel varía entre leve, moderadas o graves; generales o específicas; de corta o de larga duración y se presentan 
en la adquisición del lenguaje, lectura, escritura, razonamiento o matemáticas. Estos pueden estar dados por diversas causas como impedimentos sensoriales, retraso mental, diferencias culturales o el estudiante presenta algún problema cognitivo propio que perjudique el aprendizaje de algunas habilidades. También son resultado de problemas educativos o ambientales que se deben a las malas estrategias de enseñanza que pueden afectar el nivel del niño.

Con respecto a las Dificultades en el Aprendizaje de las Matemáticas (DAM), se pueden manifestar de diversas formas tales como acalculia, discalculia, dislexia, dificultades del procesamiento visual, dificultades de atención, concentración, etc. Pueden ser en algunos casos debidos a TDA, TDAH o por fragilidad o inmadurez atencional (Fernández, 2013). Las DAM pueden tener su origen relacionadas con el estudiante en sí dado a las diversas circunstancias por el que atraviesa o también pueden estar influenciadas por circunstancias externas, como la propia naturaleza de las matemáticas o quizás debido a la metodología de enseñanza y actitud del docente frente a la enseñanza (Carrillo, 2009). Teniendo en cuenta los antecedentes ya mencionados surgió la presente investigación, considerando uno de los factores de las DAM, correspondiente a los métodos de enseñanza y las actitudes por parte de los estudiantes o docentes. Asimismo, es importante recalcar que el principal objetivo de la enseñanza de las matemáticas no es sólo que los niños aprendan las tradicionales reglas aritméticas, las unidades de medida y las nociones geométricas, sino, su principal finalidad es que puedan resolver problemas y aplicar los conceptos y habilidades matemáticas en su quehacer diario (Ramírez, 2016).

González, Adriana y Weinstein, Edith (2005), refieren que los niños(as) utilizan los constantemente los números en situaciones de su vida diaria para formar parte de una sociedad en la cual los números están presentes en varias de las acciones que realiza el ser humano.

Por su parte Ainsworth (2006) señala, que los beneficios de hacer uso de las representaciones se logran después de que un estudiante comprende en la forma en el que presenta la codificación de la información en su representación, y cuál sería su relación con el dominio que representa. Para poder realizar esta tarea, es dable que los estudiantes demanden seleccionar una representación apropiada para ellos, o construir una, lo se convertirá en parte de un ejercicio cognitivo. 
El génesis de la investigación tiene sus inicios en la identificación de las necesidades educativas observadas en un grupo de alumnos del tercer grado de educación primaria de la Institución Educativa Cleofé Arévalo del Águila del distrito de la Banda de Shilcayo, quienes en algunos casos presentan dificultades de aprendizaje teórico práctico en el área curricular de lógico matemático específicamente en los contenidos y capacidades referidas al cálculo y numeración. Por lo cual se platea la pregunta de investigación que busca ser resuelta. ¿Los estudiantes de $3^{\circ}$ de primaria de la I.E. Cleofé A. del Águila del Dpto? de la B. Sh., mejorarán su competencia matemática en cálculo y numeración luego de la aplicación del programa de intervención psicopedagógico de orientación cognitivo? Asimismo, se justifica en la búsqueda trascendencia académica, debido a que será considerada como un antecedente para futuras investigaciones sobre la importancia la creación y ejecución responsable de los programas psicopedagógicos en la educación del niño, del mismo modo, los resultados de esta investigación servirán para constituir un alcance en la estimulación de la capacidad matemática en las fases de cálculo y numeración.

\section{ESTRATEGIAS METODOLÓGICAS O MATERIALES Y MÉTODOS}

El tipo de investigación es experimental, con diseño pre experimental de pre-test/pos-test con un solo grupo, porque pretende demostrar el efecto de la aplicación de un programa psicopedagógico de orientación cognitiva sobre un grupo experimental conformado por alumnos de tercer grado de primaria. (Hernández, Fernández, y Baptista, 2010) La población estuvo conformada por 133 estudiantes en 4 secciones de tercer grado de primaria, para lo cual se consignaron criterios de inclusión y exclusión y se logró obtener una muestra de 50 estudiantes, los mismos que fueron seleccionados de acuerdo a la calificación del curso de matemática, posteriormente, se elaboró un test de evaluación con contenido de cálculo y numeración, constituido de 13 ítems con respuestas múltiples, 9 ítems con contenido de Cálculo y 4 ítem con contenido de numeración.

El Programa y test fueron de elaboración propia y se denominó "Programa Psicopedagógico de Orientación Cognitivo", de 14 sesiones educativas las cuales tuvieron como finalidad fortalecer las competencias matemáticas, con 5 sesiones de introducción referente a capacidades cognitivas (atención, concentración, memoria y lenguaje), posterior a ello se centran en la competencia matemática en los contenidos de cálculo (problemas aditivos, problemas multiplicativos, problemas de varias etapas, 
patrón aditivo, patrón multiplicativo) y contenido de numeración (comparación, sistema de numeración). El programa busca fortalecer la competencia matemática de los alumnos en los contenidos de cálculo y numeración, para lo cual el desarrollo del programa se realiza de manera vivencial participativo y de manera lúdica, dado que la temática planteada en cada sesión de aprendizaje se basa en ejercicios de la vida cotidiana. Por otro lado, el pre test como el programa, fueron sometidos a una validación por criterio de jueces, quienes concluyeron que los ítems propuestos del test y el contenido del programa psicopedagógico son consistentes con lo que se pretende medir. Respecto a la confiabilidad del pre test se utilizó el alfa de Cronbach con un resultado de 0,8824 el cual permite señalar que el instrumento es confiable.

Por otro lado, para la aplicación del programa, se solicitó la autorización por escrito del director de la institución, así como el consentimiento y compromiso firmado de los padres de familias y/o apoderados. Se dio a conocer el alcance de la investigación a los docentes.

\section{RESULTADOS Y DISCUSIÓN}

\subsection{Descripción de los resultados}

Tabla 1 Resultados pre test de la competencia matemática de los educandos de $3^{\circ}$ de primaria de la I.E. Cleofé A. del Águila del Dtto. de la B. Sh

\begin{tabular}{lccccc}
\hline $\begin{array}{c}\text { Competencia } \\
\text { matemática }\end{array}$ & $\begin{array}{c}\text { Nro. } \\
\text { Ítems }\end{array}$ & $\begin{array}{c}\text { Puntaje } \\
\text { máximo }\end{array}$ & $\begin{array}{c}\text { Frecuencia } \\
\text { Punt. Prom. }\end{array}$ & Porcentaje & $\begin{array}{c}\text { Porcentaje } \\
\text { Acumulado }\end{array}$ \\
\hline Cálculo & 9 & 14 & 6.69 & 82.80 & 82.80 \\
Numeración & 4 & 6 & 1.39 & 17.20 & 100.00 \\
Total & 13 & 20 & 8.08 & 100.00 & \\
\hline
\end{tabular}

Fuente: Elaboración propia

Gráfico 1 Resultados pre test de la competencia matemática de los educandos de $3^{\circ}$ de primaria de la I.E. Cleofé A. del Águila del Dtto. de la B. Sh

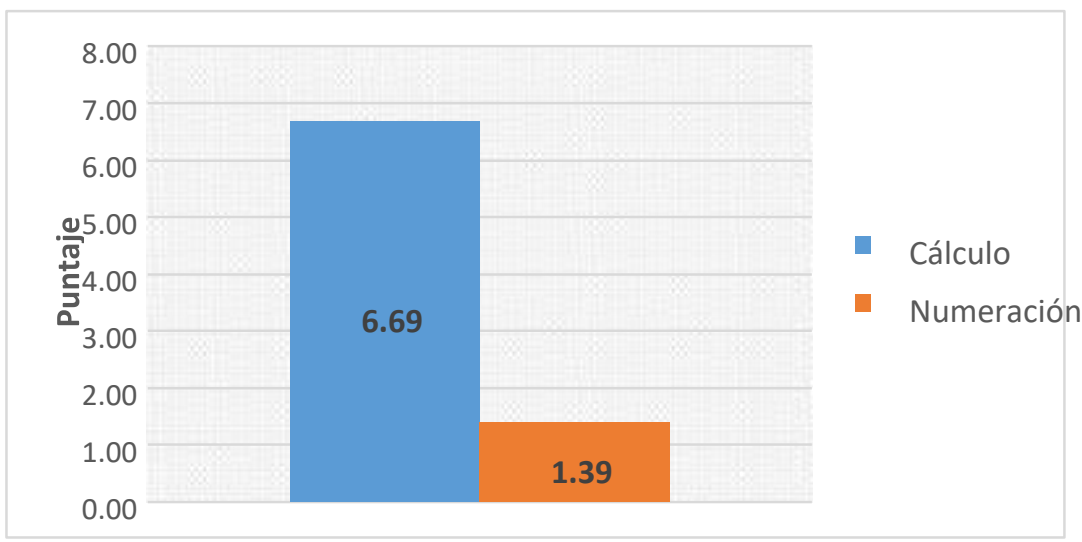

Figura 1. Puntaje pretest de la competitividad matemática 
Los resultados evidenciados en la tabla 1, gráfico 1 de acuerdo a la evaluación del pretest muestran un nivel bajo de desempeño en la competitividad matemática en las extensiones de cálculo y numeración, asimismo, se puede resaltar que estos resultados son propios del criterio de selección (estudiantes con bajo nivel de logro o desempeño) de la muestra

Tabla 2 Resultados postest de la competencia matemática de los educandos de $3^{\circ} \mathrm{de}$ primaria de la I.E. Cleofé A. del Águila del Dtto. de la B. Sh.

\begin{tabular}{lccccc}
\hline $\begin{array}{c}\text { Competencia } \\
\text { matemática }\end{array}$ & $\begin{array}{c}\text { Nro. } \\
\text { Ítems }\end{array}$ & $\begin{array}{c}\text { Puntaje } \\
\text { máximo }\end{array}$ & $\begin{array}{c}\text { Frecuencia Punt. } \\
\text { Prom. }\end{array}$ & Porcentaje & $\begin{array}{c}\text { Porcentaje } \\
\text { Acumulado }\end{array}$ \\
\hline Cálculo & 9 & 14 & 8.88 & 70.03 & 70.03 \\
Numeración & 4 & 6 & 3.80 & 29.97 & 100.00 \\
Total & 13 & 20 & 12.68 & 100.00 & \\
\hline
\end{tabular}

Fuente: Elaboración propia

\section{Gráfico 2.}

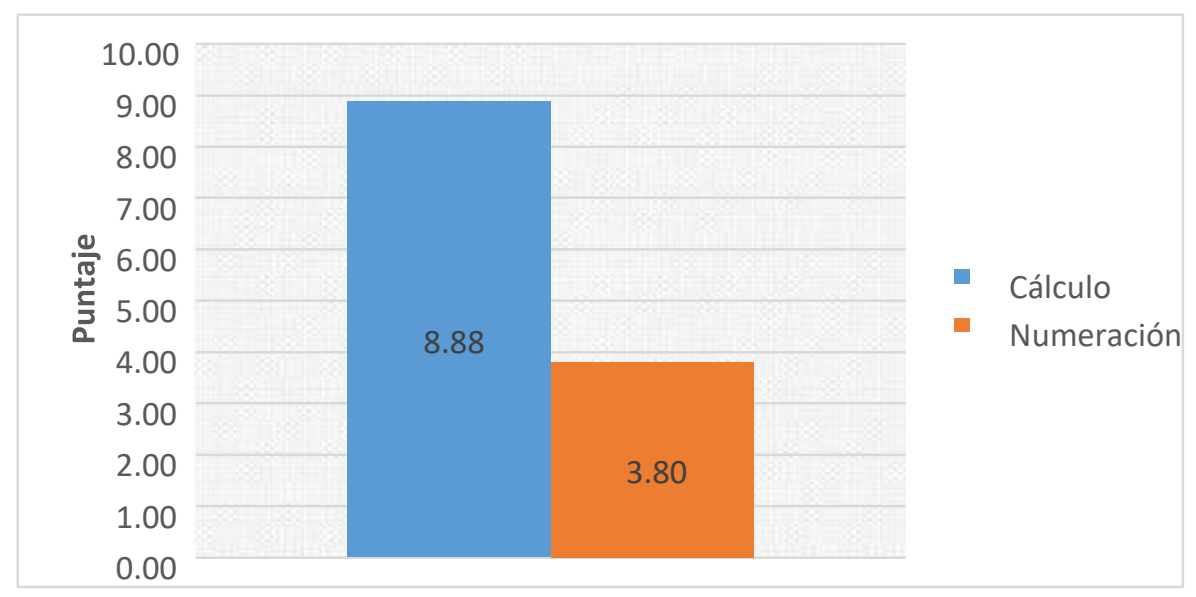

Figura 2. Puntaje postest de la competencia matemática

Posterior a la ejecución del programa e intervención psicopedagógica en orientación cognitivo se obtuvo una mejora en los efectos, así se determina en la Tabla 5 que el puntaje promedio de la competitividad matemática en los segmentos de cálculo y numeración ascendió a 8.88 y 3.80, respectivamente, conformando un promedio general de 12.68. Evidentemente se puede visualizar una mejora en los resultados.

\subsection{Discusión de resultados}

Se demuestra que el programa de intervención psicopedagógico tiene una consecuencia efectiva en la dimensión de cálculo de la variable competencia matemática. Este resultado se complementa con la investigación de Fernández (2013) en su tesis concluye que el 
$54 \%$ de los docentes considera que los orígenes primordiales de las DAM son debido a la formación y estrategia de la enseñanza y el $38 \%$ se debe a elementos cognitivos del estudiante, un $4 \%$ considera que podrían ser debidas a la naturaleza de las Matemáticas y solo el $4 \%$ a las actitudes y creencias del estudiante.

Los escolares del $3^{\circ}$ de primaria de la I.E. Cleofé A. del Águila mejoraron su competitividad matemática en el segmento de numeración. Este resultado del contraste de esta investigación presenta similitud con la investigación cuasi experimental de Chumbes (2010) quien pretende demostrar el efecto del programa JOBAS concluye que existe mejora en el grupo control después de la aplicación del programa. Al igual que con la investigación realizada, en ambos casos queda demostrado que posterior a la aplicación de un programa los alumnos mejoran significativamente sus resultados.

Queda demostrado la eficacia del programa psicopedagógico en la competencia matemática, este resultado es apoyado con la investigación de diseño pre experimental de Vásquez (2010), quién a través de su investigación demostró que, 1 conjunto con 37 colaboradores entre 7 y 8 años, del $2^{\circ}$ de primaria de nivel estatus económico formativa bajo de Ventanilla, Callao mostraron una diferencia demostrativa entre el antes y después de la aplicación del programa, esto queda demostrado mediante el ensayo no paramétrica de Wilcon que consigue un valor de 0.000 en los 4 contenidos con una elevación de significado de 0.05 . También se puede reconocer que la Dirección central por medio del MINEDU implementando esquemas para el mejoramiento del nivel académico de los niños en el curso de matemática, eso quiere decir que los va preparando para las dificultades matemáticas que se presentan en su vida diaria como lo menciona Murillo, E. (2013) en donde menciona que el nivel o provecho ilustrado de los individuos depende mucho de las técnicas de estudio utilizado por los maestros entre ellos se puede destacar que en el últimos años se han registrado capacitación docente utilizando las últimas técnicas de enseñanza en el aprendizaje lógico matemática (cálculo y numeración) los cuales no se han implementado de forma adecuada debido a la cantidad de enseñanza que hoy en día tienen los maestros para la ejecución y desarrollo de las clases, en las cuales dejan de lado la didáctica empleada en las capacitaciones.

\section{CONCLUSIÓN O CONSIDERACIONES FINALES}

El programa de intervención psicopedagógico de orientación cognitivo es eficaz en la mejora de la competencia matemática en cálculo y numeración de los alumnos del tercer 
grado de primaria.

El nivel de competencia matemática en los contenidos de cálculo y numeración antes de la aplicación del programa psicopedagógico muestran una puntuación de 8.08, y una mejora de 4.60 puntos después de la aplicación del programa psicopedagógico, obteniendo una puntuación de 12.68 .

Se evidencia que los alumnos del tercer grado de primaria mejoran significativamente su competencia matemática en la dimensión de cálculo y numeración después de la aplicación del programa de intervención psicopedagógico.

\section{LISTA DE REFERENCIAS}

Ainsworth, S. (2006) Conceptual framework for considering learning with multiple representations, Learning and Instruction: 16 183-198.

Carrillo, B. (2009). Dificultades en el aprendizaje matemático. Revista Digital Innovación y Experiencia Educativa. Recuperado de https://archivos.csif.es/archivos/andalucia/ensenanza/revistas/csicsif/revista/pdf/ Numero_16/BEATRIZ_CARRILLO_2.pdf.

Cerda, G., Pérez, C., Ortega, R. Lleujo, M. \& Sanhueza, L. (2011). Fortalecimiento de competencias matemáticas tempranas en preescolares, un estudio chileno. Psychology, Society, \& Education. Vol.3 ( $\left.\mathrm{N}^{\circ} 1\right)$, pp. 23-39.

Chumbes, J. C. (2010). Efecto del programa Juego de las Operaciones Básicas de Adición y Sustracción (JOBAS) en el nivel del cálculo mental de las operaciones básicas de los alumnos de primer grado de educación primaria de la I.E. Reino de Suecia. (Tesis de licenciatura). Universidad Femenina del Sagrado Corazón. Perú.

D’Amore, B., J. Godino y M. Fandiño (2008) Competencias y matemática, Bogotá, Magisterio.

Fernández, C. (2013). Principales dificultades en el aprendizaje de las Matemáticas. Pautas para maestros de Educación Primaria. (Tesis de pregrado). Universidad Internacional de La Rioja, Barcelona.

García Quiroga, B., Coronado, A., \& Montealegre Quintana, L. (2011). Formación y desarrollo de competencias matemáticas: una perspectiva teórica en la didáctica de las matemáticas. Revista Educación Y Pedagogía,23(59), 159- 
Programa de orientación psicopedagógico de...

175.Recuperado

https://revistas.udea.edu.co/index.php/revistaeyp/article/view/8715

Gascón, J., 1998, “Evolución de la didáctica de las matemáticas como disciplina científica", Revista Recherches en Didactique des Mathématiques, vol. 18/1, núm. 52, pp. 7-33.

González, A. \& Weinstein, E. (2005). El número y la serie numérica. En curso de formación y actualización profesional para el personal docente de educación preescolar. Volumen I, pp. 249 257. México: Secretaría de Educación Pública Recuperado de http://www.reformapreescolar.sep.gob.mx/pdf/volumen_1.pdf

Hernández, R., Fernández, C., y Baptista, M. (2010). Metodología de la investigación. México: McGraw-Hill.

León, V., Lucano, V., y Oliva, J. (2014). Elaboración y aplicación de un programa de estimulación de la competencia matemática para niños de primer grado de un colegio nacional. (Tesis inédita de postgrado). Pontificia Universidad Católica del Perú. Lima.

Ministerio de Educación. (2016). Marco de fundamentación de las pruebas de la evaluación censal de estudiantes. Recuperado de http://umc.minedu.gob.pe/wpcontent/uploads/2017/02/Marco-de-Fundamentaci\%C3\%B3n-ECE.pdf

Ministerio de Educación. (2017). El Perú en PISA 2015. Informe nacional de resultados. Lima: Oficina de Medición de la Calidad de los Aprendizajes.

Murillo, E. (2013). Factores que inciden en el rendimiento académico en el área de matemáticas de los estudiantes de noveno grado en los centros de educación básica de la ciudad de Tela, Atlántida. [Tesis inédita de postgrado, Universidad Pedagógica Nacional Francisco Morazán. Honduras] Repositorio virtual Miguel de Cervantes.

Organización para la Cooperación y el Desarrollo Económico. (2006). Pisa 2006 Marco de la Evaluación. Conocimientos y habilidades en ciencias, matemáticas y lectura. Recuperado de http://www.oecd.org/pisa/39732471.pdf

Ortega, E. (2014). Programa de intervención para solucionar las dificultades de aprendizaje de la lectura en tres alumnos de $3^{\circ}$ grado de primaria. [Proyecto de tesis de pregrado, Universidad Pedagógica Nacional] Repositorio virtual Universidad Pedagógica Nacional. 
Ramírez, C. (2016). Programa "Fácil aprendo matemática" sobre el aprendizaje de la matemática en estudiantes de $3^{\circ}$ grado de educación primaria de la I.E Fe y Alegría No 13 Collique - Comas UGEL 04 - 2015. (Tesis de postgrado). Universidad César Vallejo. Lima, Perú.

Rodríguez, C. (2014). La Importancia del Pensamiento Lógico-Matemático. Recuperado de https://educayaprende.com/la-importancia-del-pensamiento-logicomatematico/

Ruiz, Y. (2011). Aprendizaje de las matemáticas. Revista digital para profesionales de la enseñanza. Recuperado de https://www.feandalucia.ccoo.es/andalucia/docu/p5sd8451.pdf

Tobón, S. (2008). Formación basada en competencias en la educación superior. Recuperado de https://cmapspublic3.ihmc.us/rid=1LVT9TXFX1VKC0TM16YT/Formaci\%C3\%B3n\%20basada\%20en\%20competencias\%20(S ergio\%2 0Tob\%C3\%B3n).pdf

Vásquez, M. (2010). Efecto del programa "Matemática para todos" en el logro de aprendizajes en matemática de alumnos de primaria - Ventanilla. (Tesis de maestría). Universidad San Ignacio de Loyola. Lima. 